\title{
Next-to-leading order multi-leg processes for the Large Hadron Collider
}

\section{Thomas Binoth*, Thomas Reiter ${ }^{\dagger}$}

School of Physics, The University of Edinburgh, Edinburgh EH9 3JZ, UK.

E-mail: binotheph.ed.ac.uk, thomas.reiter@ed.ac.uk

\section{Jeppe R. Andersen}

CERN, CH-1211 Geneva, Switzerland.

E-mail: jeppe.andersen@cern.ch

\section{Gudrun Heinrich, Jennifer M. Smillie}

IPPP, University of Durham, Durham DH1 3LE, UK.

E-mail: gudrun.heinrich@durham.ac.uk, j.m.smillie@durham.ac.uk

\section{Jean-Philippe Guillet, Gregory Sanguinetti}

LAPTH, 9, Chemin de Bellevue BP 110, 74941 Annecy le Vieux, France.

E-mail: guillet@lapp.in2p3.fr, sangui@lapp.in2p3.fr

\section{Stefan Karg}

Institute for Theoretical Physics E, RWTH Aachen, D-52056 Aachen, Germany.

E-mail: karg@physik.rwth-aachen.de

\section{Nikolas Kauer}

Institute for Theoretical Physics, University of Würzburg, D-97074 Würzburg, Germany.

E-mail: kauer@physik.uni-wuerzburg.de

In this talk we discuss recent progress concerning precise predictions for the LHC. We give a status report of three applications of our method to deal with multi-leg one-loop amplitudes: The interference term of Higgs production by gluon- and weak boson fusion to order $\mathscr{O}\left(\alpha^{2} \alpha_{s}^{3}\right)$ and the next-to-leading order corrections to the two processes $p p \rightarrow Z Z$ jet and $u \bar{u} \rightarrow d \bar{d} s \bar{s}$. The latter is a subprocess of the four jet cross section at the LHC.

8th International Symposium on Radiative Corrections (RADCOR)

October 1-5 2007

Florence, Italy

\footnotetext{
*Speaker.

$\dagger$ This work was supported by the British Science and Technology Facilities Council (STFC), the Scottish Universities Physics Alliance (SUPA) and the Deutsche Forschungsgemeinschaft (DFG, BI-1050/1).
} 


\section{Introduction}

The Large Hadron Collider (LHC) at CERN will probe our understanding of electroweak symmetry breaking and explore physics in the $\mathrm{TeV}$ region. A detailed theoretical knowledge of various kinds of Standard Model backgrounds is indispensable for these studies. Especially in the startup-phase when it is necessary to calibrate detectors using multi-jet signatures, preferably with identifiable leptons, Standard Model processes will play an important role. Precise predictions for such multi-partonic cross sections are only possible by including higher order corrections such that renormalisation and factorisation scale dependencies are tamed. While corrections at next-toleading order in the strong coupling constant $\alpha_{s}$ are known to basically all relevant $2 \rightarrow 2$ processes, the situation for $2 \rightarrow N$ processes whith $N \geq 3$ is less satisfactory, although tremendous progress has been made in the last few years. For $2 \rightarrow 3$ processes, various methods have been used recently to obtain NLO QCD predictions for multi-boson production $p p \rightarrow Z Z Z, W W Z, H H H[1,2,3,4]$, processes in the context of weak boson fusion, like $p p \rightarrow W W j j, W Z j j[5,6], p p \rightarrow H j j$ with effective gluon-Higgs couplings [7], $g g \rightarrow H q \bar{q}$ [8], and $p p \rightarrow t \bar{t} j$ [9].

Many different techniques are used for the evaluation of multi-particle processes, as a result of recent developments which were triggered by the observation that the standard Passarino-Veltman reduction in general does not lead to numerically stable amplitude representations for the reduction of five-point integrals. Apart from Feynman diagrammatic approaches which apply new reduction techniques for some or all scalar and tensor integrals $[10,11,12]$ the evaluation of one loop amplitudes by extracting the coefficients of a certain basis set of scalar integrals using unitarity based methods [13] both in algebraic [14, 15, 16] and numerical[17, 18, 19] variants has seen substantial progress recently. Purely numerical approaches based on Feynman diagrams, which do not use any reduction to "basis integrals" are also viable [1, 20, 21, 22].

In [11] we have proposed a framework for the evaluation of one-loop multi-leg amplitudes, based on reduction formulas in Feynman parameter space $[23,24]$ in the context of dimensional regularisation. The reduction of rank $R N$-point to rank $R-1(N-1)$-point integrals can be obtained algebraically for $N>5$. For $N \leq 5$ we provide form factor representations which are expressed in terms of 3- and 4-point scalar integrals with Feynman parameters in the numerator. The dimensionality of the box functions is such that all IR divergences, i.e. poles in $1 /(n-4)$, are isolated into the triangle functions. For all IR divergent triangle functions explicit representations can be obtained. We have coded all formulas up to $N=6$ for massless internal kinematics into a FORTRAN 90 code, called golem 90 [32]. The code allows to switch between a semi-numerical and completely numerical evaluation of the basis functions. The latter is preferable in exceptional phase space regions where certain basis integrals can become linearly dependent. In this way the problems of instabilities due to inverse Gram determinants can be tamed. Optionally, our reduction formalism also allows a reduction to a scalar integral basis of 1-, 2-, 3-point functions in $n$ dimensions and 4-point functions in $n+2$ dimensions, denoted by $I_{1}^{n}, I_{2}^{n}, I_{3}^{n} I_{4}^{n+2}$. This fully algebraic reduction is to be used away from exceptional phase space points, where it is fast and reliable.

In the following we will discuss three applications of our method relevant for LHC phenomenology. 

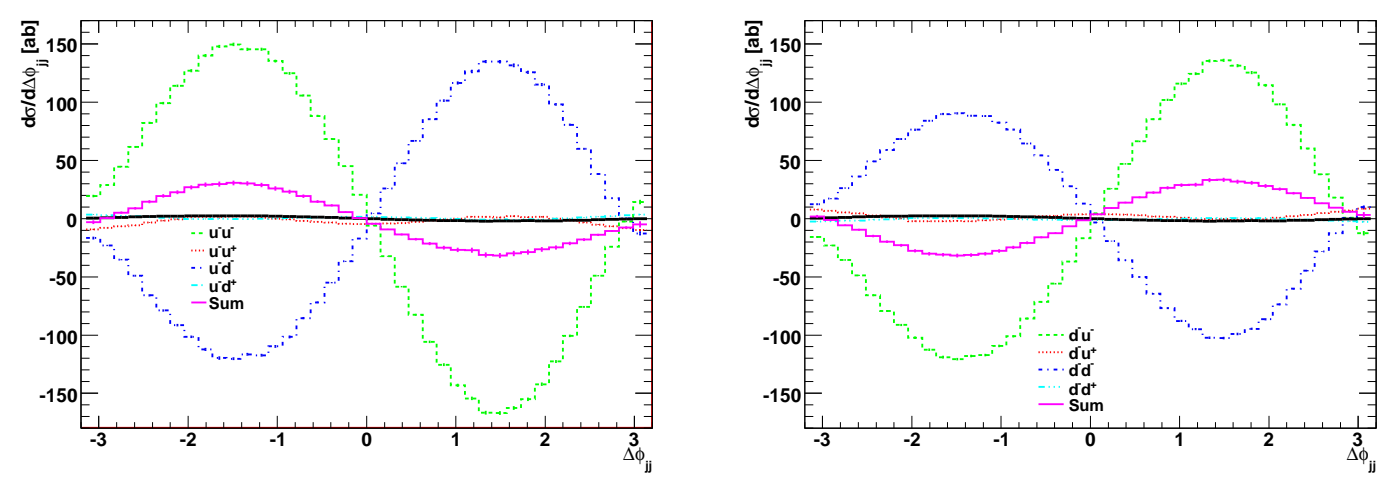

Figure 1: The $\Delta \phi_{j j}$-distribution for various flavour and helicity-configurations. The purple histogram labelled "Sum" indicates the sum over the four contributions shown. The sum over all flavour and helicity assignments including all sea flavours is shown in the black histogram. WBF cuts have been used [27].

\section{Interference term for $p p \rightarrow H j j$ at order $\mathscr{O}\left(\alpha^{2} \alpha_{s}^{3}\right)$}

Weak boson fusion is one of the most promising discovery channels for the Higgs boson. As such it deserves a careful consideration of higher order effects. Very recently, the electroweak corrections, including a recalculation of QCD corrections have been evaluated in [25]. A Born level interference term between the gluon fusion and weak boson fusion is only allowed by colour conservation if the in- and outgoing quarks are crossed in the $t \leftrightarrow u$-channel, which is kinematically disfavoured. Such interference terms are included in the calculations of [26, 25]. However, the exchange of an extra gluon between the quark lines opens up a viable colour channel. We have obtained a fully analytic result of this one-loop interference term between gluon fusion and weak boson fusion [27], and implemented the evaluation in a flexible $\mathrm{C}++$ Monte Carlo programme.

Figure 1 displays the contribution to the distribution in $\Delta \phi_{j j}$ from the interference terms for various helicity and flavour configurations for a Higgs boson mass of $115 \mathrm{GeV}$.

Note that the integral of the absolute value of the $\Delta \phi_{j j}$ distribution,

$$
\int_{-\pi}^{\pi} d \Delta \phi_{j j}\left|\frac{d \sigma}{d \Delta \phi_{j j}}\right|
$$

is a useful measure of the impact of the interference effect on the extraction of the $Z Z H$-vertex. This integral evaluates to $9.1 \mathrm{ab}$. The total integral over the absolute value of the fully differential cross section leads to $29.6 \mathrm{ab}$. We conclude that the interference term can be safely neglected in phenomenological studies, but this needed to be checked by doing the explicit calculation.

\section{The virtual $\mathscr{O}\left(\alpha_{s}\right)$ corrections to $p p \rightarrow Z Z j$}

During the Les Houches 2005 workshop the process $p p \rightarrow V V j$ was identified as one of the most important missing NLO calculations [28]. The process with a charged vector boson pair has been evaluated very recently by two independent groups [29, 30]. The evaluation for ZZ plus jet is still missing. The process is composed of three partonic reactions

$$
q \bar{q} \rightarrow Z Z g, g q \rightarrow Z Z q, g \bar{q} \rightarrow Z Z \bar{q}
$$



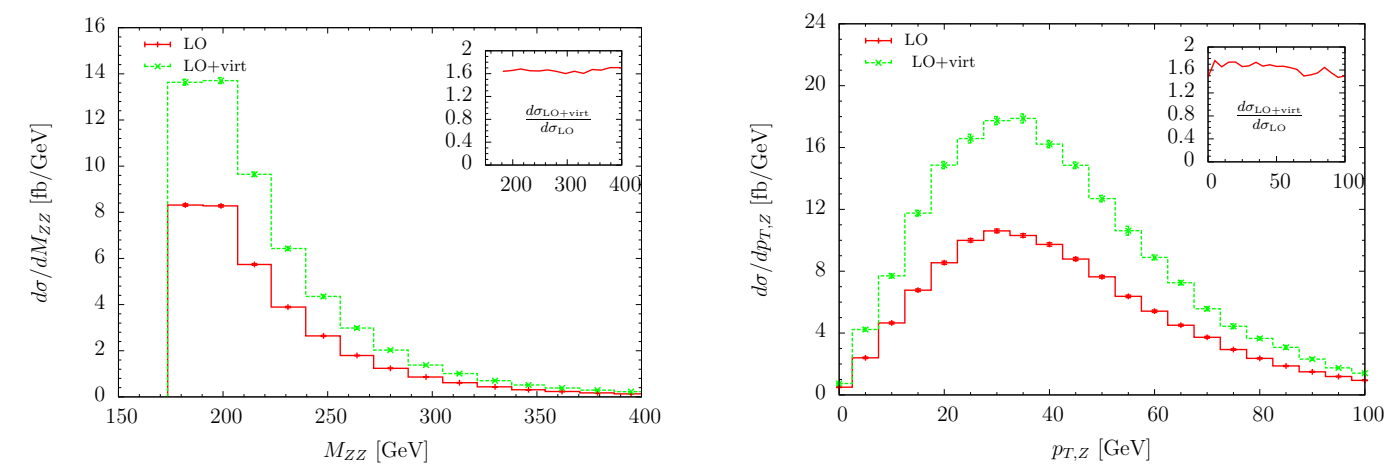

Figure 2: The finite virtual NLO contribution to the helicity component --+++ of the partonic process $q \bar{q} \rightarrow Z Z g$. The invariant mass of the $\mathrm{Z}$ pair is shown on the left and the $p_{T}$ distribution on the right. We use the cut $p_{T, \text { jet }}>20 \mathrm{GeV}$ and a parton and beam pipe separation cut of $\theta_{i j}>1.5^{0}$.

With our methods we have obtained the virtual order $\mathscr{O}\left(\alpha_{s}\right)$ corrections for all helicity amplitudes of both processes. Using spinor helicity methods we have obtained analytical formulas for the coefficients of all basis scalar integrals. We work in dimensional regularisation and treat $\gamma_{5}$ by applying the 't Hooft-Veltman scheme. As an illustration we show the contribution of the virtual correction to some typical distributions. Only the contributions which are related to finite basis integrals are plotted. For the full result the real emission corrections remain to be included [31].

\section{The amplitude $u \bar{u} \rightarrow d \bar{d} s \bar{s}$}

Not a single NLO $2 \rightarrow 4$ process relevant for LHC phenomenology has been evaluated so far. As a test case for our reduction methods we have evaluated the 6-photon amplitude [15] and have compared our result with an evaluation using a fully numerical approach [21] and unitarity based methods $[18,16]$. The same set-up can be used to attack now processes like $p p \rightarrow j j j j$ and $p p \rightarrow b \bar{b} b \bar{b}$ which are of relevance for background studies at the LHC. As an example we show the result of one colour factor of the finite virtual NLO contribution to the $u \bar{u} \rightarrow d \bar{d} s \bar{s}$ amplitude in massless QCD. The calculation has been carried out using spinor helicity amplitudes in the 't Hooft-Veltman scheme. We have chosen a convenient colour basis, which allows to split the amplitude as follows

$$
\sum_{\lambda} \sum_{i=1}^{6} \mathbf{C}^{i} A_{i}^{\lambda}\left(p_{1}, \ldots, p_{6}\right),
$$

where $A_{c}^{\lambda}$ are the helicity and colour sub-amplitudes. In particular we chose the colour structures

$$
\overrightarrow{\mathbf{C}}=\left(\delta_{c_{1}}^{c_{2}} \delta_{c_{4}}^{c_{3}} \delta_{c_{6}}^{c_{5}}, \delta_{c_{1}}^{c_{2}} \delta_{c_{4}}^{c_{5}} \delta_{c_{6}}^{c_{3}}, \delta_{c_{1}}^{c_{5}} \delta_{c_{4}}^{c_{2}} \delta_{c_{6}}^{c_{3}}, \delta_{c_{1}}^{c_{5}} \delta_{c_{4}}^{c_{3}} \delta_{c_{6}}^{c_{2}}, \delta_{c_{1}}^{c_{3}} \delta_{c_{4}}^{c_{5}} \delta_{c_{6}}^{c_{2}}, \delta_{c_{1}}^{c_{3}} \delta_{c_{4}}^{c_{2}} \delta_{c_{6}}^{c_{5}}\right)
$$

In our notation $\lambda$ is the vector $\left(\lambda_{1}, \ldots, \lambda_{6}\right)$, and $\lambda_{j}= \pm 1$ is the helicity of the particle with momentum $p_{j}$ of which the colour index is $c_{j}$. In the six-quark amplitude one can identify two independent helicity configurations, $\lambda^{a}=(+,+,+,+,+,+)$ and $\lambda^{b}=(+,+,+,+,-,-)$.

We reduced the tensor integrals to form factors as outlined above (for more details see [11]), and deal with the spinor algebra by completing spinor lines to traces. The expressions for the 


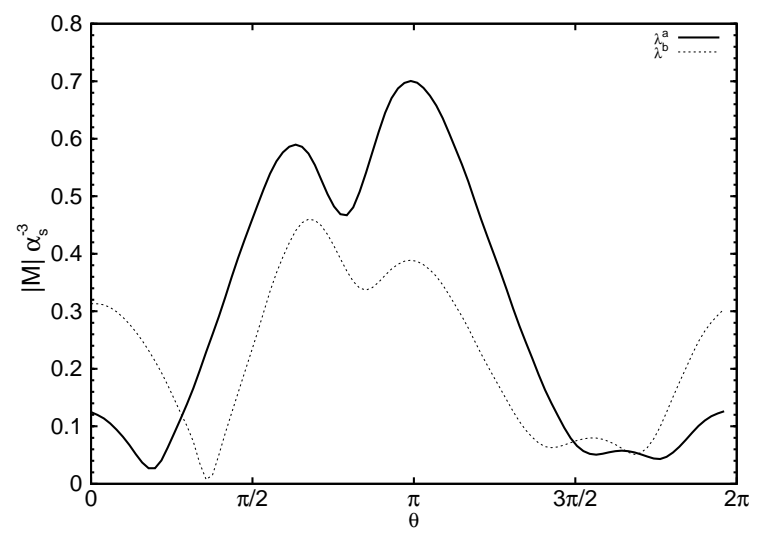

Figure 3: Six-quark amplitude. The finite parts of the two helicity configurations $s\left|A_{1}^{\lambda^{a}}\right| \alpha_{s}^{-3}$ (solid) and $s\left|A_{1}^{\lambda^{b}}\right| \alpha_{s}^{-3}$ (dashed) are plotted for an arbitrary kinematical point where the final state momenta have been rotated about the $y$-axis by an angle $\theta$.

diagrams are transformed into a Fortran 90 program. The golem 90 library is used for the numerical evaluation of the form factors. The code returns the sub-amplitudes in the form

$$
A_{i}^{\lambda}\left(p_{1}, \ldots, p_{6}\right)=\frac{g_{s}^{6}}{4 \pi^{2}} \frac{1}{s}\left(\frac{A}{\varepsilon^{2}}+\frac{B}{\varepsilon}+C+\mathscr{O}(\varepsilon)\right)
$$

for each of the six colour structures and for all non-zero helicities, where $A, B$ and $C$ are complex coefficients. As an example in Fig. 3 we plot the amplitude $s\left|A_{c}^{\lambda}\right| \alpha_{s}^{-3}$ for the colour structure $c=1$ and the two helicity configurations $\lambda^{a}$ and $\lambda^{b}$. The initial state momenta have been fixed to be aligned with the $z$-axis while the final state momenta have been rotated about the $y$-axis by an angle $\theta$. For $\theta=0$ the momenta are chosen as in Ref. [21]. In the chosen units the renormalisation scale is $\mu=1$. The amplitude has been evaluated at 50 successive points between $\theta=0$ and $\theta=2 \pi$, which took 2.4 seconds per point and helicity on an Intel Pentium $4 \mathrm{CPU}(3.2 \mathrm{GHz})$.

\section{Conclusion}

The LHC demands next-to-leading order precision for many multi-particle processes. We have presented in this talk some recent results using our one-loop reduction methods, ranging from completely massless amplitudes to ones with several mass scales. The progress which has been made in the last years in the context of precision phenomenology by many groups is good news for the LHC. Many relevant predictions beyond leading order are or will become available in the near future and there is a clear movement towards largely automated NLO tools as well as combining NLO amplitudes with parton showers.

\section{References}

[1] A. Lazopoulos, K. Melnikov and F. Petriello, Phys. Rev. D 76 (2007) 014001 [arXiv:hep-ph/0703273].

[2] V. Hankele and D. Zeppenfeld, arXiv:0712.3544 [hep-ph]. 
[3] T. Plehn and M. Rauch, Phys. Rev. D 72 (2005) 053008 [arXiv:hep-ph/0507321].

[4] T. Binoth, S. Karg, N. Kauer and R. Ruckl, Phys. Rev. D 74 (2006) 113008 [arXiv:hep-ph/0608057].

[5] B. Jager, C. Oleari and D. Zeppenfeld, JHEP 0607 (2006) 015 [arXiv:hep-ph/0603177].

[6] G. Bozzi, B. Jager, C. Oleari and D. Zeppenfeld, Phys. Rev. D 75 (2007) 073004 [arXiv:hep-ph/0701105].

[7] J. M. Campbell, R. K. Ellis and G. Zanderighi, JHEP 0610 (2006) 028 [arXiv:hep-ph/0608194].

[8] M. M. Weber, Nucl. Phys. Proc. Suppl. 160 (2006) 200.

[9] S. Dittmaier, P. Uwer and S. Weinzierl, Phys. Rev. Lett. 98 (2007) 262002 [arXiv:hep-ph/0703120].

[10] W. T. Giele and E. W. N. Glover, JHEP 0404 (2004) 029 [arXiv:hep-ph/0402152].

[11] T. Binoth, J. P. Guillet, G. Heinrich, E. Pilon and C. Schubert, JHEP 0510 (2005) 015 [arXiv:hep-ph/0504267].

[12] A. Denner and S. Dittmaier, Nucl. Phys. B 734 (2006) 62 [arXiv:hep-ph/0509141].

[13] Z. Bern, L. J. Dixon and D. A. Kosower, Annals Phys. 322 (2007) 1587 [arXiv:0704.2798 [hep-ph]].

[14] R. Britto, B. Feng and P. Mastrolia, Phys. Rev. D 73 (2006) 105004 [arXiv:hep-ph/0602178].

[15] T. Binoth, G. Heinrich, T. Gehrmann and P. Mastrolia, Phys. Lett. B 649 (2007) 422 [arXiv:hep-ph/0703311].

[16] C. Bernicot and J. P. Guillet, arXiv:0711.4713 [hep-ph].

[17] G. Ossola, C. G. Papadopoulos and R. Pittau, Nucl. Phys. B 763 (2007) 147 [arXiv:hep-ph/0609007].

[18] G. Ossola, C. G. Papadopoulos and R. Pittau, JHEP 0707 (2007) 085 [arXiv:0704.1271 [hep-ph]].

[19] R. K. Ellis, W. T. Giele and Z. Kunszt, arXiv:0708.2398 [hep-ph].

[20] C. Anastasiou, S. Beerli and A. Daleo, JHEP 0705 (2007) 071 [arXiv:hep-ph/0703282].

[21] Z. Nagy and D. E. Soper, Phys. Rev. D 74 (2006) 093006 [arXiv:hep-ph/0610028].

[22] A. Ferroglia, M. Passera, G. Passarino and S. Uccirati, Nucl. Phys. B 650 (2003) 162 [arXiv:hep-ph/0209219].

[23] Z. Bern, L. J. Dixon and D. A. Kosower, Nucl. Phys. B 412 (1994) 751 [arXiv:hep-ph/9306240].

[24] T. Binoth, J. P. Guillet and G. Heinrich, Nucl. Phys. B 572 (2000) 361 [arXiv:hep-ph/9911342].

[25] M. Ciccolini, A. Denner and S. Dittmaier, arXiv:0710.4749 [hep-ph].

[26] J. R. Andersen and J. M. Smillie, Phys. Rev. D 75, 037301 (2007) [arXiv:hep-ph/0611281].

[27] J. R. Andersen, T. Binoth, G. Heinrich and J. M. Smillie, arXiv:0709.3513 [hep-ph].

[28] C. Buttar et al., arXiv:hep-ph/0604120.

[29] S. Dittmaier, S. Kallweit and P. Uwer, arXiv:0710.1577 [hep-ph].

[30] J. M. Campbell, R. K. Ellis and G. Zanderighi, arXiv:0710.1832 [hep-ph].

[31] T. Binoth, J.-Ph. Guillet, S. Karg, N. Kauer, G. Sanguinetti; in preparation.

[32] T. Binoth, J.-Ph. Guillet, G. Heinrich, T. Reiter; in preparation. 